As part of the $23^{\text {rd }}$ ACM Computer-Chess Championship a panel discussion will be held on Tuesday, February $16^{\text {th }}, 1993$ from 3:30-5:00 p.m. in the Hyatt Hotel, under the title Computer Chess: What Remains? Panelists:

- Professor T.A. Marsland (chair) is from the University of Alberta, Edmonton, Canada. He is a longstanding member of the computer-chess community and has written extensively on tree-searching methods.

- Dr. R. Hyatt is at the University of Alabama, Birmingham. He has worked for many years developing the supercomputer version of the Cray Blitz chess program, which was Computer World Champion 19831989.

- Dr. R. Levinson is at the University of California at Santa Cruz. He is principal investigator in the "Morph" learning project.

- Professor M. Newborn is at McGill University, Montreal, Canada. He too is an established member of the computer-chess community. Developer of the first working parallel chess program, and active organizer of North American computer-chess events.

- J. Stanback is at HP Labs in Fort Cóllins. He is author of the highly successful ZARKOV chess program for small computers.

Further it is intended that either Murray Campbell or "CB" Hsu also hope to be able to serve as a panelist.

- Dr. F-h Hsu and Dr. M. Campbell are both at IBM Yorktown Heights. They are the main components in the design and testing of Deep Thought, the 1989-1992 Computer World Champion Chess Program currently undergoing major hardware revision.

\title{
Theme
}

The panelists will review the state of computer chess as the best programs begin to match world champion standards. Although it is difficult to judge when programs will be superior (estimates range from 5 to 25 years), there is a sense of inevitability. This raises several questions like: What else has been accomplished by this work? What were the spin-offs for Artificial Intelligence? What other applications have benefited from the new searching methods, and data structures? And also questions like: What remains to be done? What could be done better? How to approach related domains like GO, which are less amenable to existing search techniques? This is an opportunity forr the audience to come primed with their favorite questions and to elicit expert responses from the panelists.

\section{IBM DEEP BLUE}

\section{The Editorial Board}

In the December 1992 issue of the Danish national chess magazine Skakbladet, it was announced that "IBM Deep Blue" will be presented to the world in Copenhagen, Denmark, on February 24-27, 1993. Moreover, it was stated that a four-games match between IBM Deep Blue and IGM Bent Larsen was scheduled, with playing times from 11 am to $5 \mathrm{pm}$.

From the IBM research team, Dr. Feng-hsiung Hsu gave the following comment on this announcement:

"The machine playing is not really the final Deep Blue, but Deep Thought II running the Deep Blue preliminary software/search algorithms. There are three stages of development for Deep Blue: the software simulation on DT II (Deep Blue Simulation), the preliminary version on a prototype 10-processor machine (Baby Deep Blue), and the final 1024-processor machine (Deep Blue). Only the first-stage machine will be ready for the match. The main difference between Deep Blue Simulation and DT 2 is the introduction of new search-extensions algorithms. The Deep Blue technical presentation itself will be mainly on the new Deep Blue custom VLSI single-chip chess machine.

As for the match itself, I am not sure whether it is a 3-game or a 4-game match. The machine is supposed to play against Swedish GM Andersson or Cramling on alternate days as well." 